Table 1. Comparison between GOHAl index scores in RA, SLE and control groups

\begin{tabular}{lcccccc}
\hline & RA n=60 & SLE $\mathrm{n}=\mathbf{3 2}$ & $\begin{array}{c}\text { Controls } \\
\mathrm{n}=\mathbf{4 6}\end{array}$ & & \\
& & Median (SD) & Median (SD) & Median (SD) RA vs RA and Three \\
SLE controls groups
\end{tabular}

RA: Rheumatoid Arthritis, SLE: Systemic Lupus Erythematosus

Conclusion: RA patients presented an increased alteration in oral health perspective when compared to SLE and controls. This association demonstrates the relationship between RA and periodontitis pathogenesis.

Importance in diagnosing periodontal disorders in rheumatic diseases should be performed due to its influence in quality of life and perpetuation of an inflammatory state and oral symptoms.

References:

[1] Blaizot, A., Monsarrat, P., Constantin, A., Vergnes, J. N., de Grado, G. F., Nabet, C., ... \& Sixou, M. (2013). Oral health-related quality of life among outpatients with rheumatoid arthritis. International dental journal, 63(3), 145-153.

Disclosure of Interests: None declared

DOI: 10.1136/annrheumdis-2020-eular.5741

\section{AB1328-HPR INCREASED BODY MASS INDEX AFFECTS SPINAL MOBILITY RATHER THAN DISEASE ACTIVITY IN ANKYLOSING SPONDYLITIS}

M. He ${ }^{1}$, Y. Bao ${ }^{1}$, Y. Yang ${ }^{1}$, S. Liu', C. Dong ${ }^{1}$, W. Zhou', J. Guo ${ }^{1}$, J. Liu', Y. Chen ${ }^{1}$, Z. Gu ${ }^{1}{ }^{1}$ Affiliated Hospital of Nantong University, Nantong, China

Background: Studies have reported the association between overweight or obesity and ankylosing spondylitis (AS), but their relation is still unclear in China. Objectives: To explore the prevalence of overweight or obesity and its effect on other indicators and their relationships in Chinese AS patients.

Methods: Demographic and clinical variables were collected from 207 AS patients. Patients were categorized to normal $\mathrm{BMl}$ group $\left(\mathrm{BMl}<23 \mathrm{~kg} / \mathrm{m}^{2}\right)$ and overweight group $\left(\mathrm{BMI} \geq 23 \mathrm{~kg} / \mathrm{m}^{2}\right)$. We used Bath Ankylosing Spondylitis Disease Activity Index (BASDAI), Bath Ankylosing Spondylitis Functional Index (BASFI), Bath Ankylosing Spondylitis Metrology Index (BASMI), Health Assessment Questionnaire (HAQ), Ankylosing Spondylitis Quality of Life (ASQoL) and Medical Outcomes Study Short Form 36 (SF36) to evaluate disease activity, physical function, spinal mobility, functional limitation and health-related quality of life, respectively. Statistical analysis used independent $t$ test, Mann-Whitney U test, Chi-square test and Spearman's rank correlation test. Results: $56.5 \%$ (117) AS patients were overweight or obese, among which $80.3 \%$ (94) were male. In the overweight group, patients were older, more being married, and have higher BMl, higher waist circumference, higher waist-to-hip ratio, longer disease duration, higher BASMI score, higher white blood cell count (WBC), higher lymphocyte and higher platelet count compared to the non-overweight group $(P \leq 0.038)$. However, there was no distinct difference in BASDAl score between the two groups $(P=0.891)$. In the correlation analyses, gender and marital status $(P \leq 0.036)$ were correlated negatively with BMI; while age, waist circumference, waist-to-hip ratio, WBC, lymphocyte count and red blood cell $(P \leq 0.036)$ were correlated positively with BMI.

Conclusion: Overweight or obesity is common in Chinese AS patients. Increased BMI affects not disease activity but spinal mobility, which indicates that patients with high BMI are more likely to have limitations in flexion, extension, lateral bending and torsion of spine.

References:

[1] Bowness P. Hla-B27. Annu Rev Immunol. 2015;33:29-48.

[2] de Araújo TA, Mota MC, Crispim CA. Obesity and sleepiness in women with fibromyalgia. Rheumatology International. 2014;35(2):281-7.

Acknowledgments: This study was funded by Science and technology Project of Nantong City (Grant/Award Number: MSZ18217), Postgraduate Research \& Practice Innovation Program of Jiangsu Province (Grant/Award number: KYCX19_2071 and KYCX18 2410), National Natural Science Foundation of China (Grant/Award number: 81671616 and 81871278), Science and technology Project of Jiangsu Province (Grant/Award number: BE2018671) and Clinical Research Center of Stem Cells, Affiliated Hospital of Nantong University, Nantong (Grant/Award number: HS2018001).

Disclosure of Interests: None declared

DOI: 10.1136/annrheumdis-2020-eular.6213

\section{AB1329-HPR THE CURRENT STATUS AND ASSOCIATED FACTORS OF FATIGUE IN CHINESE PATIENTS WITH GOUT}

J. Guo ${ }^{1}$, W. Zhou ${ }^{1}$, M. He ${ }^{1}$, Z. Gu ${ }^{1}$, C. Dong ${ }^{1} .^{1}$ Affiliated Hospital of Nantong University, Nantong, China

Background: Fatigue of chronic diseases has been paid more and more attention. but the status of fatigue in gout patients has not been reported all the world[1].

Objectives: In the absence of previous studies, our study aims to investigate the fatigue status, explore the potential predictors of fatigue and the effects of fatigue on health-related quality of life (HRQoL) among Chinese gout patients.

Methods: This cross-sectional study was conducted from the Affiliated Hospital of Nantong University. A series of questionnaires were applied: Fatigue Scale-14 (FS-14), the $10 \mathrm{~cm}$ visual analog scale (VAS), the Patient Health Questionnaire (PHQ-9), the Generalized Anxiety Disorder questionnaire (GAD-7), the Pittsburgh Sleep Quality Index (PSQI), Health Assessment Questionnaire(HAQ), the Short Form 36 health survey (SF-36). Laboratory examinations were taken to obtain some biochemical indicators. Independent samples t-test, Mann-Whitney U-test, Chi-square analysis, Pearson /Spearman correlation, Stepwise linear regression and binary logistic regression were used to analyze the data.

Results: 411 gout patients were included in this study. Among them, more than $50 \%$ patients reported physical fatigue in $\mathrm{FS}-14$, severe disease, poor psychological status and reduced HRQoL were associated with fatigue. Multiple stepwise linear regression and binary logistic regression were applied and showed that pain, sleep quality, anxiety, depression and functional disorder were the potential predictors of fatigue. In addition, we found that the more severe the fatigue, the lower the patient's HRQoL.

Conclusion: Fatigue among gout patients is exceedingly common. The results of this study suggested that rheumatologists should pay closely attention to gout patients who suffer from serious fatigue, especially those with pain, poorer sleep quality, anxiety, depression and functional disorder.

References:

[1] Henry, A., Tourbah, A., Camus, G., Deschamps, R., Mailhan, L., Castex, C., Gout, O. \& Montreuil, M. (2019) Anxiety and depression in patients with multiple sclerosis: The mediating effects of perceived social support, Multiple sclerosis and related disorders. 27, 46-51.

Disclosure of Interests: None declared

DOI: 10.1136/annrheumdis-2020-eular.6338

\section{AB1330-HPR ASSOCIATION OF INTERSTITIAL LUNG DISEASE AND QUALITY OF LIFE IN CHINESE RHEUMATOID ARTHRITIS}

Y. Wang ${ }^{1}$, R. Zhao', C. Dong ${ }^{1}$, Z. Gu ${ }^{1} .{ }^{1}$ Affiliated Hospital of Nantong University, Nantong, China

Background: The health-related quality of life (HRQL) of patients with rheumatoid arthritis associated interstitial lung disease (RA-ILD) is less concerned(1). This study hypothesized that HRQL of such patients decreased.

Objectives: The aim of this study was to assess the difference in HRQL between RA with and without ILD, and to predict risk factors of HRQL in Chinese RA-ILD. Methods: A cross-sectional survey of RA-ILD patients included socio-demographic characteristics, clinical variables, psychological factors and HRQL. Data were analyzed by Student's t or chi-square test. Linear regression analysis was used to investigate the predictors of HRQL in RA-ILD.

Results: The data showed that $35.17 \%(51 / 145)$ of RA patients had ILD. There are significant differences in age, sex, smoking history, disease duration and activity between RA with and without ILD. HRQL of RA-ILD was impaired. Physical component score (PCS) of patients with RA-ILD was also worse than that of RA without ILD $(48.91 \pm 2.48$ vs. $50.80 \pm 2.55)$. Linear regression analysis showed that DAS28 and fatigue were risk factors for PCS in patients with RA-ILD, while depression and rural residence were risk factors for mental components summary in Table 1

Table 1. Stepwise multiple linear regression analysis of PCS and MCS in RA-ILD

\begin{tabular}{lccccc}
\hline Predictors & B & SE & $\mathbf{t}$ & $P$ & $\mathbf{9 5 \%} \mathbf{C l}$ \\
\hline PCS & & & & & \\
DAS28 & -0.777 & 0.227 & -3.425 & 0.001 & $-1.236,-0.319$ \\
Fatigue & -0.655 & 0.206 & -3.177 & 0.003 & $-1.071,-0.238$ \\
MCS & & & & & \\
Depression & 2.188 & 0.669 & 3.272 & 0.002 & $0.836,3.539$ \\
Rural residents & -1.609 & 0.756 & -2.128 & 0.040 & $-3.137,-0.081$ \\
\hline
\end{tabular}

Footnotes: $\mathrm{Cl}=$ Confidence interval. $\mathrm{PCS}=$ Physical component score; MCS=Mental component score; DAS28=28-joint disease activity score. 
Conclusion: Compared with RA without ILD, HRQL of patients with RA-ILD was significantly impaired. Disease activity, fatigue, depression and rural residence were independent predictors of HRQL in Chinese RA-ILD.

References:

[1] Zamora-Legoff JA, Krause ML, Crowson CS, Ryu JH, Matteson EL. Patterns ofinterstitial lung disease and mortality in rheumatoid arthritis. Rheumatology (Oxford, England). 2017;56(3):344-50

Disclosure of Interests: None declared

DOI: 10.1136/annrheumdis-2020-eular.6249

\section{AB1331-HPR ACTIVE DISEASE ACTIVITY IN ANKYLOSIS SPONDYLITIS: WORSE OUTCOMES AND POORER HR-QOL}

W. Zhou ${ }^{1}$, M. He ${ }^{1}$, R. Zhao ${ }^{1}$, C. Dong ${ }^{1}$, Z. Gu ${ }^{1} .{ }^{1}$ Affiliated Hospital of Nantong University, Nantong, China

Background: Ankylosing spondylitis (AS) is a chronic inflammatory disease that mainly affects the sacroiliac joints and the spine, resulting in decline in quality of life[1,2]. Poor QoL is significantly related to high disease activity[3]. However, there is no systematic report on which prognosis indicators are affected by disease activity in AS patients.

Objectives: This study aimed to evaluate the patient-reported outcome measures and health-related quality of life (HR-QoL) in AS patients defined on the basis of the Bath Spondylitis Ankylosing Disease Activity Index (BASDAI).

Methods: 204 AS patients were involved in this study. A serious of questionnaires were used to overall assess AS patients, which include: Bath Ankylosing Spondylitis Disease Activity Index (BASDAI), Bath Ankylosing Spondylitis Metrology Index (BASMI), Bath Ankylosing Spondylitis Functional Index (BASFI), the $10 \mathrm{~cm}$ Visual Analog Scale (VAS), the Self-Rating Anxiety Scale (SAS), the Self-Rating Depression Scale (SDS), the Pittsburgh Sleep Quality Index (PSQI), the Health Assessment Questionnaire-Disability Index (HAQ-DI), the Fatigue Severity Scale(FSS) and the Short Form 36 Health Survey (SF-36). Independent samples t-test, Mann-Whitney U-test, Chi-square analysis and Pearson /Spearman correlation were used to analyze the data.

Results: The results demonstrated $31.4 \%$ AS patients were in active disease activity stage. Active AS patients were older, unemployed, and had less exercise therapy than stable AS patients. Besides, AS patients with active disease activity presented more severe pain $(P<0.001)$, poor physical function $(P<0.001)$ and spinal mobility $(P<0.001)$. They were more anxious $(P<0.001)$, depressed $(P<0.001)$ and had more sleep disturbance $(P=0.001)$. Compared with active AS patients, stable AS patients had more leukocytes $(P=0.040)$, lymphocytes $(P=0.002)$, erythrocytes $(P=0.001)$ and hemoglobin $(P<0.001)$. Active disease activity had a significant impact on all dimensions of quality of life in AS patients $(P<0.001)$.

Conclusion: These findings suggested that medical personnel should pay more attention to active AS patients and make effective interventions to improve quality of life.

References:

[1] Exarchou S, Lindstrom U, Askling J, Eriksson JK, Forsblad-d'Elia H, Neovius M, Turesson C, Kristensen LE, Jacobsson LT (2015) The prevalence of clinically diagnosed ankylosing spondylitis and its clinical manifestations: a nationwide register study. Arthritis research \& therapy 17:118. doi:10.1186/ s13075-015-0627-0

[2] Qian Q, Xu X, He H, Ji H, Zhang H, Ding Y, Dai SM, Zou Y, Zhu Q, Yang C, Ye S, Jiang L, Tang JP, Tong Q, He D, Zhao D, Li Y, Ma Y, Zhou J, Yuan Z, Zhang J, Jin L, Zhou X, Reveille JD, Zou H, Wang J (2017) Clinical patterns and characteristics of ankylosing spondylitis in China. Clinical rheumatology 36 (7):1561-1568. doi:10.1007/s10067-017-3660-3

[3] Huang JC, Qian BP, Qiu Y, Wang B, Yu Y, Zhu ZZ, Hu J, Qu Z (2017) Quality of life and correlation with clinical and radiographic variables in patients with ankylosing spondylitis: a retrospective case series study. BMC musculoskeletal disorders 18 (1):352. doi:10.1186/s12891-017-1711-1

Acknowledgments: Thanks to all the authors for their efforts and thanks to all members of the Department of Rheumatology of Affiliated Hospital of Nantong University for their helpfulness in the acquisition of data.

Disclosure of Interests: None declared

DOI: 10.1136/annrheumdis-2020-eular.6288

\section{AB1332-HPR ASSESSING THE PATIENT EXPERIENCE OF LUPUS NEPHRITIS: DEVELOPMENT OF A CONCEPTUAL MODEL AND REVIEW OF EXISTING PATIENT- REPORTED OUTCOME (PRO) MEASURES}

P. Berry ${ }^{1}$, K. Burrows ${ }^{2}$, R. Hall ${ }^{2}$, A. Gater ${ }^{2}$, H. Bradley², A. Ward², C. Tolley², P. Delong ${ }^{1}$, E. C. Hsia ${ }^{3} .^{1}$ Janssen Global Services LLC, Horsham, United States of America; ${ }^{2}$ Adelphi Values Ltd, PCO, Bollington, United Kingdom; ${ }^{3} J a n s s e n$ Research \& Development LLC, Spring House, United States of America
Background: Lupus nephritis (LN) is an autoimmune disease characterized by inflammation of the kidneys as a result of systemic lupus erythematosus (SLE). Approximately $50 \%$ of SLE patients will develop LN, which is considered to be one of the most severe manifestations of SLE and the leading cause of morbidity and mortality in SLE. While there is ample existing evidence on disease experience and PROs used in extra-renal SLE, little research has been done in LN. Qualitative interviews with patients can help identify concepts that are both important and relevant to the patient. In order to effectively evaluate treatment benefit, it is critical that PRO measures used to assess such concepts and define clinical trial endpoints are fit for purpose and have strong evidence of content validity in the specific context of use.

Objectives: The objective of this study was to understand the patient experience of $L N$ and to identify and characterize the signs and symptoms of $L N$ and their impact on health-related quality of life (HRQoL) through the development of a disease-specific conceptual model. This model was then used to evaluate the content validity of existing PRO measures available for use in $\mathrm{LN}$.

Methods: A structured literature search was conducted in Medline, Embase and PsycINFO to identify qualitative research articles documenting the patient experience of LN. PRO measures developed or commonly used to assess patient experiences of LN were also identified. Semi-structured concept elicitation interviews were conducted with 15 adult patients in the US with a clinician-confirmed diagnosis of LN (defined in accordance with established clinical guidelines). Supplementary qualitative data were also collected from a review of publicly available online blogs/forums. Findings were used to inform the development of a conceptual model detailing the impact of LN signs, symptoms and HRQoL and evaluate the validity of existing measures used within $\mathrm{LN}$.

Results: Searches revealed a paucity of qualitative research conducted with LN patients, supporting the need for prospective research in LN. Consistent with existing literature in SLE, the core signs and symptoms identified from the qualitative literature review, interviews and blog/forum review included joint pain, fatigue, joint stiffness, swelling (particularly in the extremities) and skin rashes LN patients also reported urinary frequency, urgency, foamy urine and blood in their urine. Disease impact on physical functioning, activities of daily living emotions, social life, work/finances and sleep were reported. PRO measures commonly used to evaluate patient experiences in LN included the SF-36 LupusQOL, LupusPRO, SLE Symptom Checklist, KDQoL and KSQ. Conceptual mapping of instruments against the newly developed conceptual model (Fig ure 1) highlighted that no single measure provides a comprehensive assessment of all symptoms/impact important to LN patients. Furthermore, items are largely focused on impact of symptoms with few items on symptom severity.

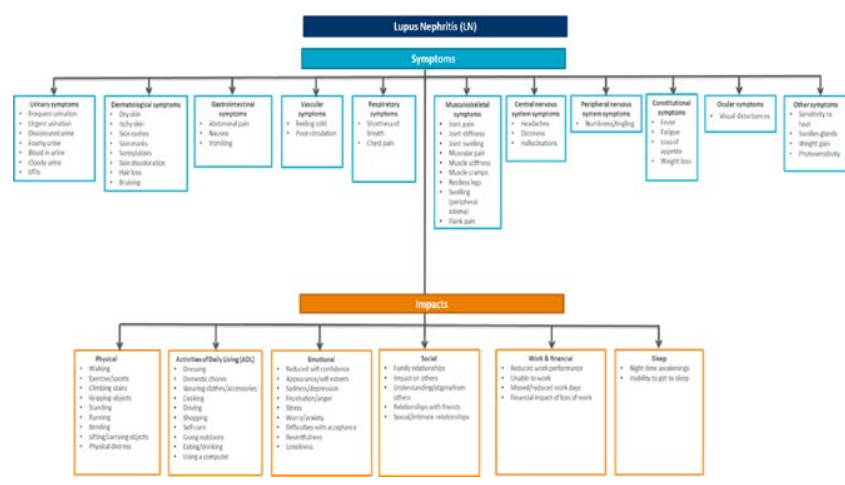

Figure 1. Conceptual model of lupus nephritis symptoms and associated impacts

Conclusion: The presentation of signs and symptoms in LN patients appears similar to those reported in extra-renal SLE populations, with the addition of swelling and urinary symptoms. Qualitative research with LN patients guided the development of a comprehensive LN conceptual model outlining the disease experience from the patients' perspective. These insights can be useful to inform PRO measurement strategies for clinical trials in LN.

Acknowledgments: With thanks to Dr. Betty Diamond and Dr. David Wofsy for their collaboration and helpful insights

Disclosure of Interests: Pamela Berry Employee of: Janssen, Kate Burrows Consultant of: Adelphi Values a health outcomes research company commissioned by Janssen to conduct the research reported in this abstract, Rebecca Hall Consultant of: Adelphi Values a health outcomes research company commissioned by Janssen to conduct the research reported in this abstract., Adam Gater Consultant of: Adelphi Values a health outcomes research company commissioned by Janssen to conduct the research reported in this abstract, Helena Bradley Consultant of: Adelphi Values a health outcomes research company commissioned by Janssen to conduct the research reported in this abstract, Amy Ward Consultant of: Adelphi Values a health outcomes research company 